\title{
Radiofrequency-assisted "reconstruction" of the right ventricular outflow tract in muscular pulmonary atresia with ventricular septal defect
}

\author{
G Hausdorf, I Schulze-Neick, P E Lange
}

\begin{abstract}
A case of pulmonary atresia with ventricular septal defect is reported in which a communication was established between the right ventricle and the hypoplastic pulmonary artery by intervention, despite muscular atresia of the right ventricular outflow tract. The atresia was perforated with a special designed radiofrequency catheter (Osypka). After the creation of a canal within the muscular atresia, balloon dilatation (diameters $2,3.5$, and $7.2 \mathrm{~mm}$ ) was performed. Arterial oxygen saturation increased from $64 \%$ to $78 \%$. Lateral deviation of the radiofrequency catheter resulting in a lateral perforation of the atretic muscular infundibulum was well tolerated without later sequelae. Early restenosis within two weeks necessitated the implantation of a stent within the "recanalised" atresia resulting in an increased anterograde flow to the pulmonary artery.

This case shows that "recanalisation" of muscular atresia of the pulmonary artery by radiofrequency is a promising technique. Additionally, stent implantation into the infundibulum to prevent restenosis is a first step to interventional right ventricular outflow tract reconstruction.
\end{abstract}

(Br Heart f 1993;69:343-346)

Pulmonary atresia with ventricular septal defect remains a therapeutic challenge for both the paediatric cardiologist and the cardiac surgeon. Staged surgical correction is the rule before definite correction can be performed. ${ }^{1-3}$ Although it has been suggested that increasing the pulmonary blood supply in pulmonary atresia is beyond the scope of interventional cardiology, Qureshi et $\mathrm{al}^{4}$ showed that transcatheter perforation of pulmonary valve atresia by a hot tip laser and subsequent balloon dilatation of the atretric "valve" is a promising technique. However, they thought that in muscular atresia interventional perforation and "recanalisation" might be impossible. ${ }^{4}$

We report on a patient with muscular pulmonary atresia and a ventricular septal defect in whom a channel was created between the right ventricle and the pulmonary artery after "recanalisation" of the atresia with a special designed ablation catheter (Osypka) and subsequent balloon dilatation and stent implantation in the "recanalised" area.

\section{Case report}

A 5 year old girl with pulmonary atresia and ventricular septal defect was referred to our hospital for interventional perforation of pulmonary valve atresia. The girl was deeply cyanotic with a body weight of $18 \mathrm{~kg}$. Echocardiographic examination showed situs solitus with normal atrioventricular connections. The aorta originated from both ventricles and overrode a large ventricular septal defect. A main pulmonary artery could not be shown echocardiographically.

At cardiac catheterisation pulmonary atresia with ventricular septal defect and two major aortopulmonary collaterals arising from the descending aorta was shown. The systemic arterial oxygen saturation was $64 \%$. One of the collateral vessels perfused parts of the left upper lobe without communicating with the pulmonary artery (no "washin" or "washout") 5 and the other aortopulmonary collateral communicated with the left pulmonary artery and a catheter could be placed in the main pulmonary artery through this communication. Simultaneous injections into the main pulmonary artery and the right ventricular infundibulum (fig 1) clearly showed muscular atresia of the pulmonary artery: the distance between the right ventricular infundibulum and the main pulmonary artery was approximately $6 \mathrm{~mm}$ (fig 1 ).

Mechanical perforation of the atresia was attempted with a special designed 0.0014 inch perforation needle with a stiff and sharpened distal end and flexible proximal part. It was unsuccessful. Then we used a special designed radiofrequency catheter (2 F, Osypka) that focused energy at its tip. A first attempt from the main pulmonary artery was unsuccessful, because the radiofrequency catheter deviated laterally and perforated the infundibulum. This, however, was well tolerated and did not cause symptoms and sequelae. However, anterograde perforation was performed successfully after we preshaped the ablation catheter manually. Perforation of the muscular atresia required energy of 8-10 Watts per second, whereas perforation of the

Herzzentrum Berlin, Gerlin, Germ I Schulze-Neick P E Lange
} 


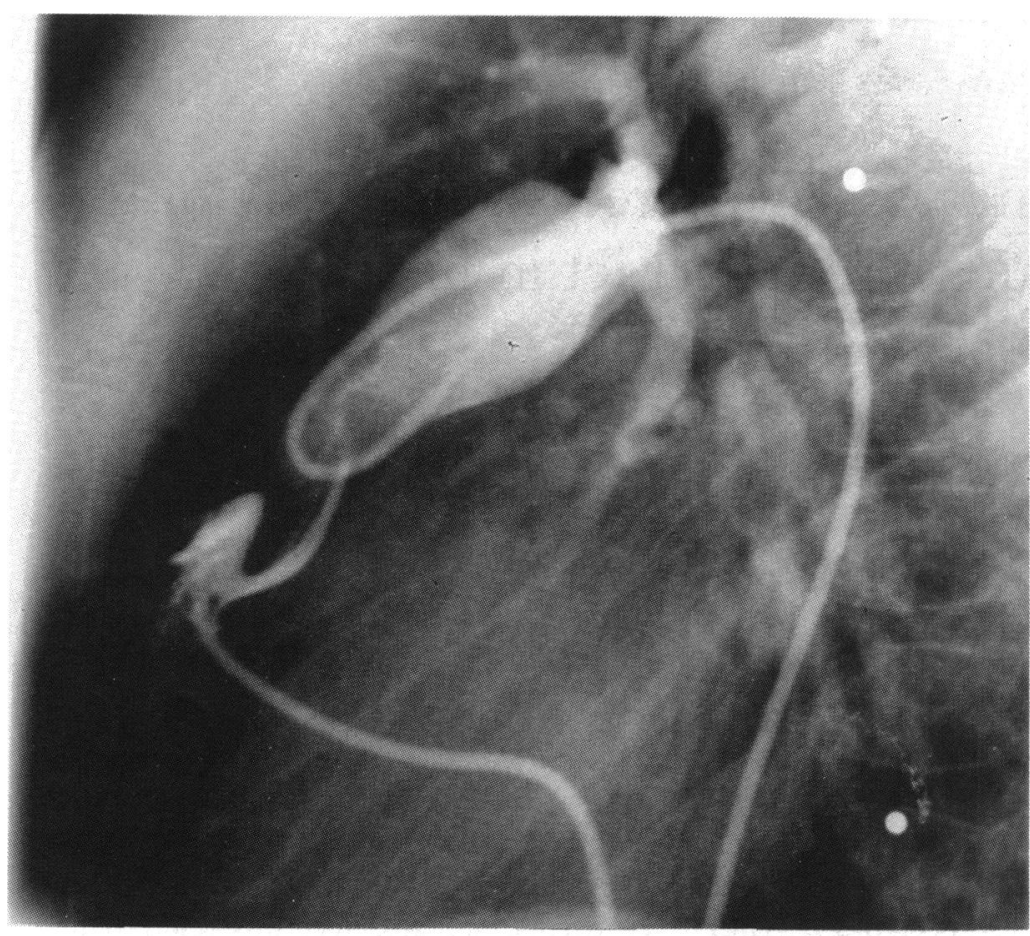

Figure 1 Angiogram obtained after simultaneous injections of contrast into the blind ending main pulmonary artery and the right ventricular infundibulum showing an area of atresia of approximately $6 \mathrm{~mm}$ between the blind end of the main pulmonary artery and the right ventricular infundibulum. This atresia of the pulmonary artery is clearly muscular.

tissue covering the blind end of the main pulmonary artery required 18 Watts per second.

After successful "recanalisation" of the muscular atresia we positioned a PTCA catheter (Orion, Cordis) with a distal diameter of $1.5 \mathrm{~F}$ and a balloon diameter $2 \mathrm{~mm}$ in the "recanalised" area and inflated the balloon. After this pre-dilatation we inflated a $3.5 \mathrm{~mm}$ balloon and then we changed the guidewire before inflating a Cordis PTA

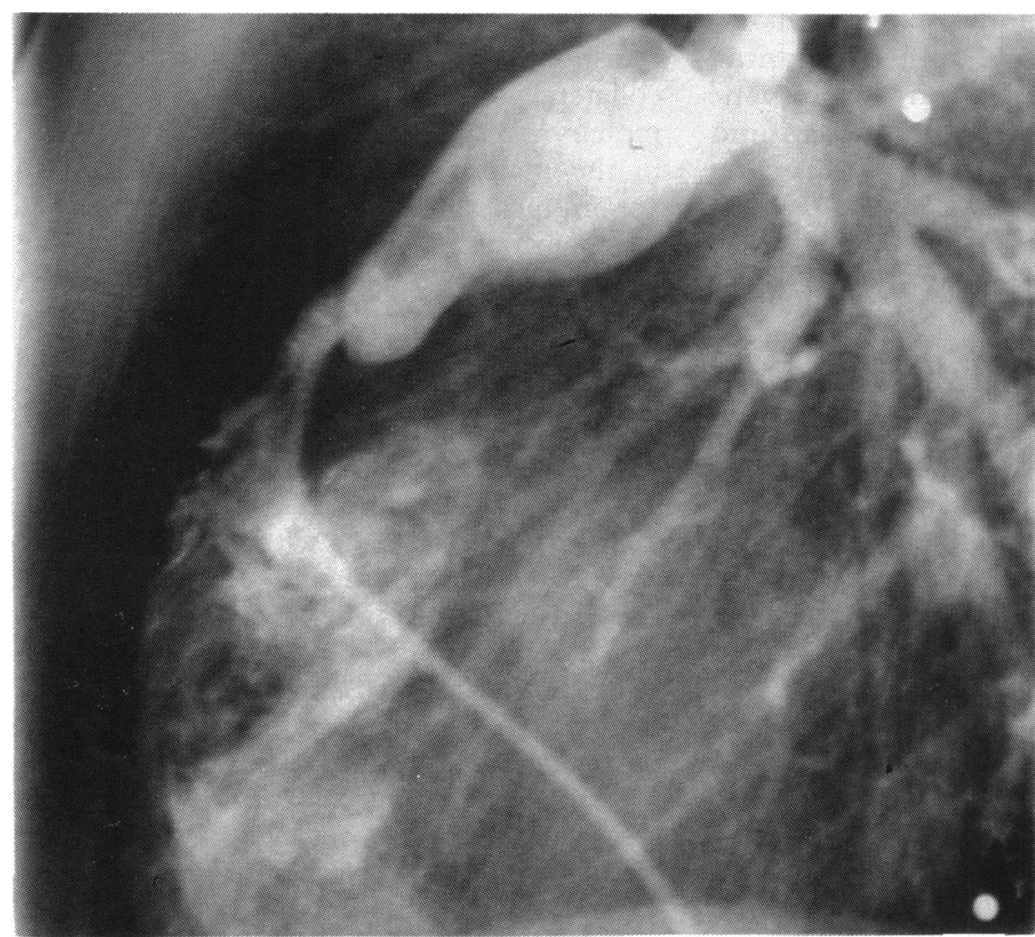

Figure 2 Angiogram obtained after perforation ("recanalisation") and balloon dilatation of the muscular atresia showing a satisfactory but slightly eccentric communication between the right ventricle and the main pulmonary artery. The systemic arterial oxygen saturation increased from $64 \%$ to $78 \%$. dilatation catheter (balloon diameter $6 \mathrm{~mm}$ at $2 \mathrm{~atm}, 7.2 \mathrm{~mm}$ at $12 \mathrm{~atm}$ ) to $12 \mathrm{~atm}$. After dilatation of the atretic region there was a satisfactory but slightly eccentric communication between the right ventricle and the main pulmonary artery (fig 2 ). The systemic arterial oxygen saturation increased to $74 \%$. Doppler echocardiography showed anterograde flow in the right ventricular outflow tract and the pulmonary artery and a gradient of $54 \mathrm{~mm} \mathrm{Hg}$ was measured. Colour coded Doppler echocardiography showed a turbulent flow with a jet width of $5 \mathrm{~mm}$. After the intervention a loud (grade 4) harsh murmur at the left sternal border developed.

Two weeks later only slight anterograde flow was shown echocardiographically and the harsh murmur was quicker. Repeat cardiac catheterisation showed significant stenosis of the "recanalised" pulmonary atresia (fig 3). A Cordis PTA-dilatation catheter (balloon diameter $6 \mathrm{~mm}$ at $2 \mathrm{~atm}, 7 \cdot 2 \mathrm{~mm}$ at $12 \mathrm{~atm}$ ) was inflated in the stenosis and showed a "waist" at the pulmonary end of the "atresia". This "waist" disappeared at an inflation pressure of $5 \mathrm{~atm}$. No extrasystoles occurred either when the balloon was inflated up to $12 \mathrm{~atm}$ for $3 \mathrm{~min}$ or when a 0.0035 inch guide wire was brought under tension in the infundibulum. We therefore decided to implant a stent into the "recanalised" atretic area to prevent restenosis.

We inserted an $8 \mathrm{~F}$ long sheath and positioned a $12 \mathrm{~mm}$ long Palmaz "iliac" stent in the opened "atresia" and expanded to $7 \mathrm{~mm}$ (fig 4). There were no extrasystoles during implantation of the Palmaz stent. Stent implantation resulted in an increased anterograde flow to the pulmonary artery and the arterial oxygen saturation increased from $64 \%$ to $78 \%$. Holter monitoring showed no extrasystoles or arrhythmias. Heparin (400 U/kg daily) was given for anticoagulation with aspirin ( $5 \mathrm{mg} / \mathrm{kg}$ daily). Dicumarol was given for 2 months. Two days after the intervention the child was sent home.

\section{Discussion}

Optimisation of pulmonary blood supply is a first step to correct pulmonary atresia with ventricular septal defect. Aortopulmonary shunts and palliative reconstruction of the right ventricular outflow tract have been performed surgically to increase pulmonary blood flow. ${ }^{367}$ Operation, however, often results in kinking and distortion of the pulmonary arteries. ${ }^{389}$ Postoperative scar formation complicates later correction and increases the surgical risk. Thus, there may be advantages to an interventional approach to pulmonary atresia with ventricular septal defect.

Qureshi et $a l^{4}$ reported successful transcatheter perforation of pulmonary valve atresia. However, they did not consider that interventional cardiology would be a suitable treatment for muscular atresia of the pulmonary artery. Our case shows that "recanalisation" of muscular atresia by means of 


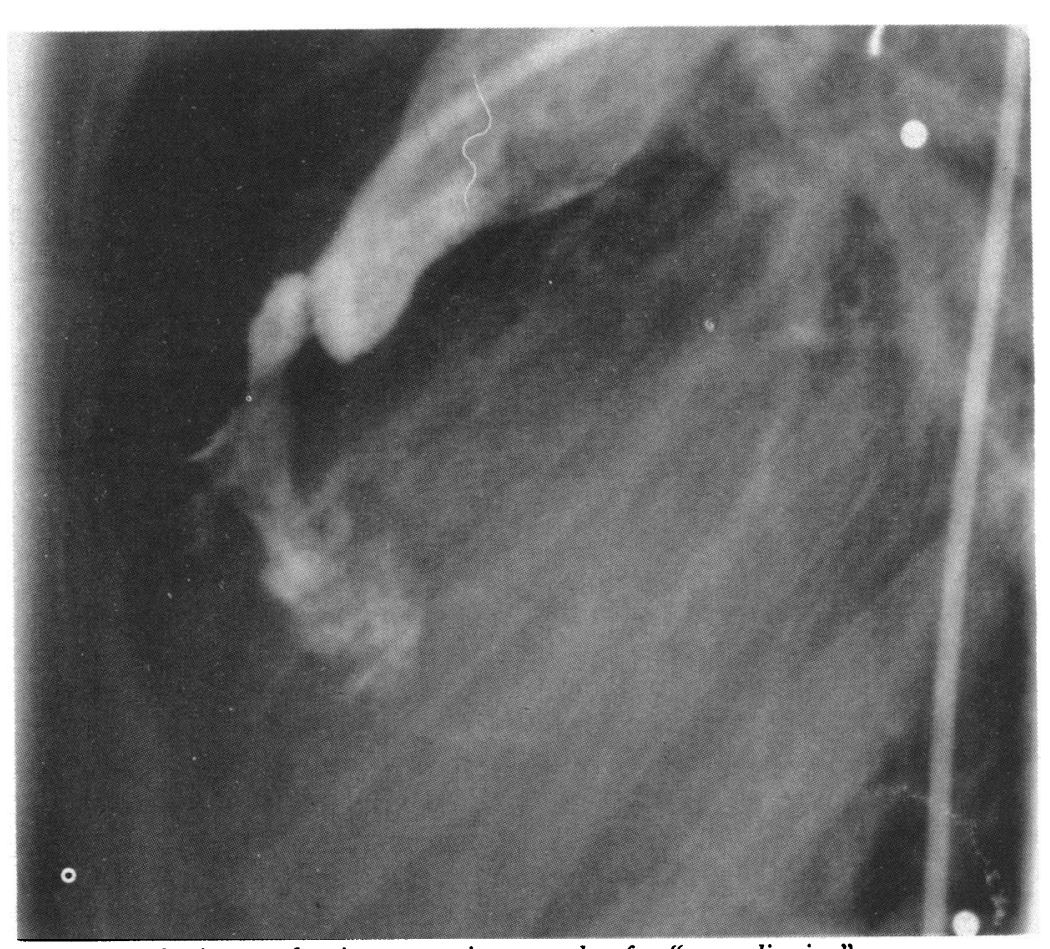

Figure 3 Angiogram showing restenosis two weeks after "recanalisation".

interventional cardiology is possible. Technically, it is important that inadvertent perforation of the free wall due to lateral deviation of the radiofrequency catheter was well tolerated. The radiofrequency catheter is designed to focus energy at its tip so that lateral tissue damage is limited. It is interesting that less energy was required to "recanalise" the muscular atresia than to perforate the tissue covering the blind end of the main pulmonary artery.

Though continuity was established through

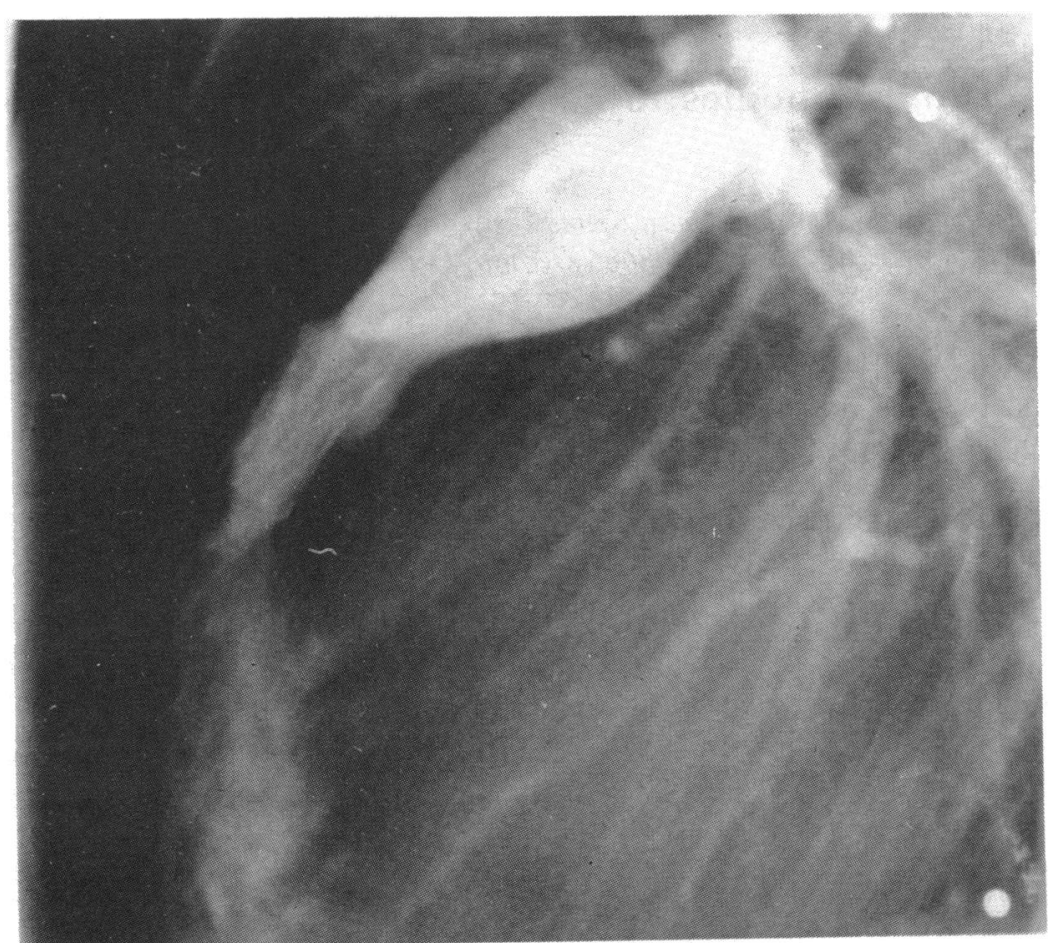

Figure 4 After re-dilatation of the recanalised atresia a $12 \mathrm{~mm}$ long Palmaz "iliac" stent was positioned in the opened "atresia" and expanded to $7 \mathrm{~mm}$. There were no extrasystoles during implantation of the Palmaz stent. Stent implantation into the "recanalised" atresia resulted in increased anterograde flow to the pulmonary artery and an increase in arterial oxygen saturation from $64 \%$ to $78 \%$. the right ventricle and pulmonary artery by intervention but restenosis that severely restricted anterograde blood flow occurred within two weeks. The creation of an adequate communication between the right ventricle and the pulmonary artery remains difficult with this interventional approach. Overdilatation of the "recanalised" atresia with large balloons is hazardous with unpredictable short-term and long-term results. Implantation of a stent in the "recanalised" atresia seems to be a promising way of preventing restenosis and of creating a defined communication between right ventricle and pulmonary artery. Stent implantation into a muscular atresia, however, could cause important and potentially life-threatening rhythm disturbances. ${ }^{10}$ Because neither use of a guide wire nor inflation of the balloon provoked extrasystoles we performed stent implantation. This was not associated with extrasystoles or rhythm disturbances-perhaps this was because the muscular atresia contained more fibrous tissue. The arrhythmogenic potential of stent implantation, however, remains uncertain. Despite these limitations, stent implantation into a muscular atresia can be used to create a defined right ventricle-pulmonary artery continuity. This communication can be enlarged up to $20 \mathrm{~mm}$ by balloon dilatation, so in theory a graded increase of pulmonary blood flow can be achieved and pulmonary blood flow can be increased in a predictable manner. Such "reconstruction" of the right ventricular outflow tract avoids surgical distortion of the pulmonary anatomy and thereby provides optimal conditions for the growth of the pulmonary arterial tree. Pulmonary regurgitation is a matter of concern, but it is well tolerated after surgical reconstruction of the right ventricular outflow tract and may have a beneficial effect on the growth of the pulmonary arterial tree. ${ }^{289}$

This case shows that "recanalisation" of muscular atresia of the pulmonary artery by radiofrequency is a promising technique. The risk of inadvertent perforation seems to be low because little tissue damage occurs. In addition, early restenosis was prevented by stent implantation into the recanalised atretic area as a first step to interventional right ventricular outflow tract "reconstruction".

1 Iyer KS, Mee RBB. Staged repair of pulmonary atresia with ventricular septal defect and major systemic to pulmonary artery collaterals. Ann Thorac Surg 1991;51: $65-72$.

2 Gill CC, Moodie DS, McGoon DC. Staged surgical management of pulmonary atresia with diminutive pulmonary arteries. I Thorac Cardiovasc Surg 1977;73:

3 Millikan JS, Puga FJ, Danielson GK, Schaff HV, Julsrud $P R$, Mair DD. Staged surgical repair of pulmonary atresia, ventricular septal defect, and hypoplastic, confluen pulmonary arteries. $f$ Thorac Cardiovasc Surg 1986; 91:818-25.

4 Qureshi S, Rosenthal E, Tynan M, Anjos R, Baker E. Transcatheter laser-assisted balloon pulmonary valve dilation in pulmonic valve atresia. $\mathrm{Am} \mathcal{F}$ Cardiol 1991;67:428-31.

5 Fäller K, Haworth SG, Taylor JFN, Macartney FJ. Duplicate sources of pulmonary blood supply in pul- 
monary atresia with ventricular septal defect. $B r$ Heart $\mathcal{f}$ 1981;46:263-8.

6 Kirklin JW, Bargeron LM, Pacifico D. The enlargement of small pulmonary arteries by preliminary palliative operations. Circulation 1977;56:612-7.

7 Steward S, Alexson C, Manning J, Oakes D, Eberly SW. Long-term palliation with the classic Blalock-Taussig shunt. F Thorac Cardiovasc Surg 1988;96:117-21

8 Freedom RM, Pongiglione G, Williams WG, Trusler GA, Rowe RD. Palliative right ventricular outflow tract construction for patients with pulmonary atresia, ventricular septal defect, and hypoplastic pulmonary arteries. $f$ Thorac Cardiovasc Surg 1983:86:24-36.

9 Piehler JM, Danielson GK, McGoon DC, Wallace RB, Fulton RE, Mair DD. Management of pulmonary atreFulton RE, Mair DD. Management of pulmonary atresia with ventricular septal defect and hypoplastic pulmonary arteries by right ventricular outflow construction. $\mathcal{F}$ Thorac Cardiovasc Surg 1980;80:552-67. 10 O'Laughlin MP, Perry SB, Lock JE, Mullins CE. Use of endovascular stents in congenital heart disease. Circulation 1991;83:1923-39.

\title{
A new marker for atherosclerosis?
}

Heat shock or stress proteins are generally thought to be cardioprotective. Several, including hsp65, show increased expression in the cells of human atherosclerotic plaques, where they may be acting as molecular chaperones.

However, $\mathrm{Xu}$ et al have shown that antibodies to hsp65 are independently associated with carotid atherosclerosis in the over $60 \mathrm{~s}$. What are the origins of these antibodies and do they play a part in the pathogenesis of atherosclerosis? It may be that the antibodies are produced in response to human hsp65 expressed by stressed cells during the early stages of atherosclerotic plaque formation.
This would suggest that atherogenesis has an autoimmune component. In individuals with atherosclerosis antibodies to hsp65 might represent a physiological immune regulatory phenomenon, or the antibodies may be pathological-directed against the plaque cells. There is a further possibility. Because the stress proteins are phylogenetically well preserved, the antibodies may have arisen against bacterial hsp65, implying an infective aetiology for atherosclerosis.

Whatever their origin and function, such antibodies may turn out to be a useful marker of atherosclerosis.

S A THORNE

\section{Association of serum antibodies to heat-shock protein 65 with carotid atherosclerosis}

\author{
Qingbo Xu, Johann Willeit, Miklos Marosi, Roman Kleindienst, \\ Friedrich Oberhollenzer, Stefan Kiechl, Thomas Stulnig, Gerhard Luef, Georg Wick
}

\begin{abstract}
Arteriosclerotic lesions can be induced in normocholesterolaemic rabbits by immunisation with heat-shock protein (hsp) 65, a stress protein expressed in high concentrations in human atherosclerotic lesions. If an immune reaction to hsp 65 also plays a part in human atherogenesis, it should be possible to detect anti-hsp 65 antibodies in patients with atherosclerotic lesions.

To study the possible relation between immune reaction to hsp 65 and atherosclerosis, 867 normal inhabitants of South Tyrol, aged 40-79 years, were selected randomly for determination of serum antibodies against hsp65, simultaneous sonographic assessment of carotid atherosclerotic lesions, and evaluation of established risk factors-ie, blood cholesterol, hypertension, smoking, diabetes
\end{abstract}

mellitus, and obesity. Autoantibodies to nuclear antigens, thyroid antigens, and rheumatoid factors were also measured. Serum anti-hsp65 antibodies were significantly $(p<0.05)$ increased in subjects aged $60-79$ years with carotid atherosclerosis compared with those without lesions, and increased antibody concentration was independent of age, sex, and other established risk factors. On the other hand, the incidence and titres of autoantibodies did not correlate with carotid atherosclerotic lesions.

Our data provide the first evidence of a strong correlation between anti-hsp 65 antibodies and carotid atherosclerosis, suggesting that hsp65 might be involved in the pathogenesis of atherosclerosis. (Lancet 1993;341:255-59.) 